\title{
Weyl and Lidskil inequalities for general hyperbolic polynomials
}

\author{
Denis Serre \\ École Normale Supérieure \\ de Lyon* \\ January 16, 2008
}

\begin{abstract}
The roots of hyperbolic polynomials satisfy the linear inequalities that were previously established for the eigenvalues of Hermitian matrices, after a conjecture by A. Horn. Among them are the so-called Weyl and Lidskiu inequalities. We give an elementary proof of the latter for hyperbolic polynomials. This proof is free from representation theory and Schubert calculus arguments, as well as from hyperbolic partial differential equations theory.
\end{abstract}

Notations: We deal with $n$-uplets of real numbers, indexed by $\{1, \ldots, n\}$. The set $\mathcal{I}_{r}^{n}$ of parts $I \subset\{1, \ldots, n\}$ of cardinality $r$ has itself cardinality

$$
\left(\begin{array}{c}
n \\
r
\end{array}\right)=\frac{n !}{r !(n-r) !}
$$

We order $\mathcal{I}_{r}^{n}$ in the following natural way. Each element $I \in \mathcal{I}_{r}^{n}$ consists in indices $(1 \leq) i_{1}<$ $\cdots<i_{r}(\leq n)$. We say that $I \prec J$ if $i_{1} \leq j_{1}, \ldots, i_{r} \leq j_{r}$, and we say that $I$ is lower than $J$, or that $J$ is higher than $I$. Given two elements $I, K$ in $\mathcal{I}_{r}^{n}$, we form the segment $[I, K]$ of elements $J \in \mathcal{I}_{r}^{n}$ such that $I \prec J \prec K$; it is void unless $I \prec K$. Likewise, $(I, K],[I, K)$ and $(I, K)$ are the segments where we require additionally that either $J \neq I$ or $J \neq K$ or both. Although $\prec$ is not a total order, the class $\mathcal{I}_{r}^{n}$ has a lowest element $\min _{r}=\{1, \ldots, r\}$ and a highest one $\max _{r}=\{n-r+1, \ldots, n\}$. Unless ambiguity, we simply write min and $\max$ for $\min _{r}$ and $\max _{r}$. The "open" segment $(\min , \max )$ also has a lowest element supmin $:=\{1, \ldots, r-1, r+1\}$ and a highest one submax $:=\{n-r, n-r+2, \ldots, n\}$. We define the order-reversing involution $\pi$ over $\{1, \ldots, n\}$ by $\pi j:=n+1-j ; \pi$ acts likewise on $\mathcal{I}_{r}^{n}$ by

$$
\pi\left\{i_{1}, \ldots, i_{r}\right\}:=\left\{n-i_{r}+1, \ldots, n-i_{1}+1\right\} .
$$

*UMPA (UMR 5669 CNRS), ENS de Lyon, 46, allée d'Italie, F-69364 Lyon, cedex 07, FRANCE. 
For instance, we have $\pi \min =\max$ and $\pi$ supmin $=$ submax.

In this paper, we deal with ordered families of $n$ real-valued continuous functions $v \mapsto$ $\lambda_{1}(v) \leq \cdots \leq \lambda_{n}(v)$ defined over a real vector space $V$. If $I \subset\{1, \ldots, n\}$ is a set of indices, we denote

$$
\Lambda_{I}(v):=\sum_{i \in I} \lambda_{i}(v)
$$

If $I \prec J$, we have $\Lambda_{I}(v) \leq \Lambda_{J}(v)$.

\section{Introduction}

\subsection{The sum of Hermitian matrices}

We briefly recall some facts about the eigenvalues $\gamma_{1} \leq \gamma_{2} \leq \cdots \leq \gamma_{n}$ of the sum of two $n \times n$ Hermitian matrices $A$ and $B$. Denoting by $\alpha_{1} \leq \alpha_{2} \leq \cdots \leq \alpha_{n}$ the eigenvalues of $A$ and $\beta_{1} \leq \beta_{2} \leq \cdots \leq \beta_{n}$ those of $B$, the trace identity gives

$$
\gamma_{1}+\cdots+\gamma_{n}=\alpha_{1}+\cdots+\alpha_{n}+\beta_{1}+\cdots+\beta_{n} .
$$

This equality is the only one satisfied by the spectra of every triplet $(A, B, A+B)$. In $1912, \mathrm{H}$. Weyl [19] used the Rayleigh-Ritz ratio to prove the following inequalities.

$$
(k+1=i+j) \Longrightarrow\left(\gamma_{k} \geq \alpha_{i}+\beta_{j}\right) .
$$

A special case $(i=j=k=1)$ tells that the smallest eigenvalue $A \mapsto \alpha_{1}$ is a concave function. Using the fact that $\alpha_{i}(-A)=-\alpha_{n+1-i}(A)$, we also have the elementary fact that $\alpha_{n}$ is convex, which comes from its expression as the supremum of the linear forms $A \mapsto x^{*} A x$ as $x$ runs over the unit sphere.

It is not hard to verify that if $n=2$, every triple $\alpha, \beta, \gamma \in \mathbb{R}^{2}$ satisfying (1) and (2) can be realized as the spectra of three Hermitian (and even real symmetric) matrices $A, B$ and $A+B$. This is not true however when $n \geq 3$ and it turns out that the number of independent inequalities increases with $n$. In 1949, Ky Fan [4] discovered the following inequality

$$
\gamma_{1}+\cdots+\gamma_{r} \geq \alpha_{1}+\cdots+\alpha_{r}+\beta_{1}+\cdots+\beta_{r}, \quad \forall r \leq n,
$$

which tells that $A \mapsto \alpha_{1}+\cdots+\alpha_{r}$ is concave, and consequently $A \mapsto \alpha_{r+1}+\cdots+\alpha_{n}$ is convex. Then B. V. Lidskil [17] found in 1950 the necessary condition that the vector $\gamma \in \mathbb{R}^{n}$ must lie in the convex hull of vectors $\alpha+\beta^{\sigma}$ as $\sigma$ runs over the symmetric group, where

$$
\beta^{\sigma}:=\left(\beta_{\sigma(1)}, \ldots, \beta_{\sigma(n)}\right) .
$$

A few years later, H. Wielandt [20] remarked that Lidskiı's property is equivalent to the set of inequalities

$$
\sum_{i \in I} \gamma_{i} \geq \sum_{i \in I} \alpha_{i}+\sum_{i=1}^{r} \beta_{i}, \quad \forall r \leq n, \quad \forall I \subset\{1, \ldots, n\}, \operatorname{card} I=r .
$$


In short, (4) writes $\gamma_{I} \geq \alpha_{I}+\beta_{\text {min }}$, with

$$
\alpha_{I}(v):=\sum_{i \in I} \alpha_{i}, \quad \gamma_{I}(v):=\sum_{i \in I} \gamma_{i}
$$

When $I=\{1, \ldots, r\}$, this inequality reduces to Ky Fan's.

During the next decade, other inequalities were found, all having the form

$$
\sum_{k \in K} \gamma_{k} \geq \sum_{i \in I} \alpha_{i}+\sum_{j \in J} \beta_{j}
$$

for some subsets $I, J, K$ of $\{1, \ldots, n\}$ with equal cardinality $r, r<n$. For instance, Freede and Thompson obtained (5) whenever $k_{p}+p=i_{p}+j_{p}$ for every $p=1, \ldots, r$, with still the ordering $i_{1}<\cdots<i_{r}, j_{1}<\cdots<j_{r}$ and $k_{1}<\cdots<k_{r}$.

In 1962, A. Horn [9] established a large (but finite for every $n$ ) list of inequalities of the form (5) in a recursive way, and conjectured that they describe exactly the attainable set of $\gamma$ 's when $\alpha$ and $\beta$ are given. He proved this equivalence for low values of $n$, but a full proof was given only in the late nineties. For a full account of this exciting mathematical saga, where W. Fulton, A. Klyachko [11], A. Knutson and T. Tao [12] made definitive contributions, see the review articles [5] by W. Fulton and [2] by R. Bhatia.

Horn actually described sets $T_{r}^{n}$ of triples $(I, J, K)$, with $I, J, K$ in $\mathcal{I}_{r}^{n}$, satisfying the restriction (7) below. The definition of $T_{r}^{n}$ involves the knowledge of the sets $T_{s}^{r}$ for every $1 \leq s \leq r-1$. Horn conjectured that for given vectors $\alpha$ and $\beta$, all possible vectors $\gamma$ of spectra of matrices $A+B$ as above, form the polytope defined by the inequalities (5) as $(I, J, K)$ runs over $T_{r}^{n}$. There is however a gap between $T_{r}^{n}$ and the set $H_{r}^{n}$ of all inequalities of the form (5) satisfied by every sum of Hermitian matrices. When $n \geq 4$, the inequalities associated to the triples in $T_{r}^{n}$ imply other ones, actually many ones as $n$ increases.

We do not list here all of Horn's inequalities, but content ourselves to describe the triplets $(I, J, K)$ for $r=2$. They consist in indices $i_{1}<i_{2}, j_{1}<j_{2}$ and $k_{1}<k_{2}$ such that

$$
i_{1}+j_{1} \leq k_{1}+1, \quad i_{1}+j_{2} \leq k_{2}+1, \quad i_{2}+j_{1} \leq k_{2}+1, \quad i_{1}+j_{1}+i_{2}+j_{2}=k_{1}+k_{2}+3 .
$$

We point out that a repeated application of Weyl's inequalities would only give triples in $\mathcal{I}_{2}^{n}$ for which the last constraint in (6) is replaced by $i_{1}+j_{1}+i_{2}+j_{2}=k_{1}+k_{2}+2$; it is not hard to see that the replacement of the last 2 by a 3 yields an improvement, in the sense that the inequalities of the form (5) corresponding to triplets of the form (6) cannot be deduced form the inequalities of Weyl. Thus second-order Horn's inequalities are independent from first-order ones, the latter being nothing but Weyl's. Remark that the triplets satisfying (6) are independent of $n \geq 3$, provided it is larger than $k_{r}$ of course. This is a general fact that the triplets of cardinality $r$ are independent of $n>r$. Of course every admissible triplet $(I, J, K)$ gives rise to other inequalities by chosing lower indices $i^{\prime}, j^{\prime}$ while chosing higher indices $k^{\prime}$, because of the monotonicity of the sets $\alpha, \beta, \gamma$. However, we do not regard such inequalities as new ones. For instance, when $r=2$, it is enough to retain only the triplets satisfying the constraint $i_{1}+j_{1}+i_{2}+j_{2}=k_{1}+k_{2}+3$, although many triplets with $i_{1}+j_{1}+i_{2}+j_{2} \leq k_{1}+k_{2}+3$ 
are valid for (5). More generally, triplets of cardinality $r$ may not be optimal unless

$$
\sum_{i \in I} i+\sum_{j \in J} j=\sum_{k \in K} k+\frac{r(r+1)}{2} .
$$

Notice that the disjoint union of "optimal" admissible triplets is not optimal, as remarked above in the case of Weyl's triplets. We point out also that there is some redundency among "optimal" inequalities, whenever $n \geq 6$.

Horn's conjecture, now a theorem, may be split into two parts. First, the set of spectra $\gamma$, when $\alpha$ and $\beta$ are given, is a convex polytope. Second, this polytope is defined by Horn's inequalities. The first part was proved by A. H. Dooley, J. Repka and N. J. Wildberger with the help of convexity properties of the moment map of an action of a Lie group over a symplectic manifold, a result by B. Kostant, improved by M. Atiyah. The second one involves Schubert calculus and representation theory of the unitary group $U(n)$. It was achieved by the papers of A. Klyachko on the one hand, and of A. Knutson and T. Tao on the other hand.

\section{$1.2 \quad$ Hyperbolic polynomials}

Given a homogenous polynomial $P$ over a real vector space $V$ of dimension $d$ (the degree of $P$ is denoted by $n$ ), we say that $P$ is hyperbolic with respect to a vector $e$ if $P(e) \neq 0$ (usually, one normalizes $P$ by $P(e)=1$ ) and if , for every $v \in V$, the roots of the univariate polynomial $t \mapsto P(t e-v)$ are real. For a hyperbolic polynomial $P$, we denote the roots of $P(\cdot e-v)$ by

$$
\lambda_{1}(v) \leq \cdots \leq \lambda_{n}(v)
$$

counting with multiplicity. Remark that $\lambda_{i}(v+c e)=\lambda_{i}(v)+c$ and $\lambda_{i}(-v)=-\lambda_{\pi i}(v)$. The polynomial is recovered by

$$
P(v)=\prod_{i=1}^{n} \lambda_{i}(v) .
$$

The connection with Hermitian matrices is that the determinant is a hyperbolic polynomial (with respect to the identity matrix $I_{n}$ ) over $\mathbf{H}_{n}$, the space of Hermitian matrices. Its degree is $n$, while $d=n^{2}$. The roots $\lambda_{j}(A)$ are nothing but the eigenvalues of $A$.

The terminology comes from the theory of partial differential equations. Hyperbolicity was shown by Gårding [6] to be the necessary and sufficient condition for local well-posedness of the Cauchy problem

$$
P(\nabla) u=f \quad \text { in } \mathbb{R}^{d},
$$

with initial data on a hyperplane $e \cdot x=$ constant. Gårding proved a number of sound results, among them the fact that the connected component $\Gamma_{P}$ of $e$ within the complement of the characteristic cone $\mathbb{R}^{d} \backslash\{P=0\}$, is a convex cone, and $P$ is hyperbolic with respect to every element of $\Gamma_{P}$. This open set is called the forward cone of $P$. It turns out that $\Gamma_{P}$ is also the set of vectors $v$ satisfying $\lambda_{1}(v)>0$, and therefore its convexity is equivalent to the concavity of $\lambda_{1}$. Likewise, $\lambda_{n}$ is a convex function over $V$, and $v \mapsto \max \left\{\lambda_{n}(v),-\lambda_{1}(v)\right\}$ is a semi-norm, a norm 
in nondegenerate cases. The analogue of Ky Fan's results follows immediately, by considering the polynomial

$$
P^{(r)}(v):=\prod_{I \in \mathcal{I}_{r}^{n}} \Lambda_{I}(v)
$$

Since the latter is hyperbolic too: the symmetric polynomials in the numbers $\Lambda_{I}(v)$, when $I$ runs over $\mathcal{I}_{r}^{n}$, are polynomials in $\sigma_{1}(\lambda(v)):=\lambda_{1}+\cdots+\lambda_{n}, \ldots, \sigma_{n}(\lambda(v)):=\lambda_{1} \cdots \lambda_{n}$, and thus $P^{(r)}$ is a homogeneous polynomial over $V$ of degree $C_{n}^{r}$. If $t \in \mathbb{R}$, we have

$$
P^{(r)}(t e-v):=\prod_{I \in \mathcal{I}_{r}^{n}}\left(r t-\Lambda_{I}(v)\right)
$$

and the $\Lambda_{I}$ 's become the roots of $P^{(r)}$, a hyperbolic polynomial, in the normalized direction $e^{(r)}:=r^{-1} e$. The smaller root of $P^{(r)}$ is $\Lambda_{\min }$, which must be concave from Gårding's result.

An important case of hyperbolic differential operators is that of first-order operators acting on vector fields. They write

$$
L:=\sum_{i=1}^{d} A_{i} \frac{\partial}{\partial x_{i}},
$$

where the $A_{i}$ 's are $n \times n$ real or complex matrices. Let us define the symbol

$$
A(\xi):=\sum_{j} \xi_{j} A_{j}, \quad \xi \in \mathbb{R}^{d} .
$$

Well-posedness (hyperbolicity) in direction $\xi_{0} \in \mathbb{R}^{d}$ is equivalent to the fact that $A\left(\xi_{0}\right)$ is nonsingular and $A\left(\xi_{0}\right)^{-1} A(\eta)$ has real eigenvalues for every $\eta$. It is always possible to transform a first-order hyperbolic operator into an equivalent one $\tilde{L}$, the latter being hyperbolic in the direction $\tilde{\xi}_{0}=(1,0 \ldots, 0)$ and having $\tilde{A}\left(\xi_{0}\right)=I_{n}$. Then the determinant is a hyperbolic polynomial over the real vector space spanned by the matrices $A_{i}$, and its roots are the eigenvalues of the matrices $\tilde{A}(\xi)$. P. Lax [15] showed that when $n \geq 4$, the space $V$ is not conjugated to a subspace of $\mathbf{H}_{n}$ in general.

In the same paper, Lax proved the "extremal" Weyl-type inequalities. By extremal, we mean those Weyl inequalities that belong to Wielandt ones:

$$
\lambda_{k}(u+v) \geq \lambda_{k}(u)+\lambda_{1}(v) .
$$

They contain as a particular case Gårding's theorem that $\lambda_{1}$ is concave. They also tell that each root $\lambda_{k}$ is weakly contractive:

$$
\left|\lambda_{k}(v)-\lambda_{k}(u)\right| \leq\|v-u\|, \quad\|\cdot\|:=\max \left\{\lambda_{n},-\lambda_{1}\right\}
$$

Applying (8) to the polynomial $P^{(r)}$ constructed above, with $k=2, k=N-1$ and $k=N$, where $N=\left|\mathcal{I}_{r}^{n}\right|$, we also obtain inequalities of Wielandt's form (4), for $I=$ supmin, $I=$ submax and $I=$ max. However, we shall obtain below much more general results.

Lax's proof involves PDE tools, in particular the finite propagation velocity in the Cauchy problem for hyperbolic equations. In a companion paper, H. Weinberger [18] gave an alternate, 
PDE-free, proof using only classical analysis, say the Intermediate Value Theorem. This is the strategy that we use here and push forward. To begin with, we need to add an argument taken from algebraic geometry, since the proof in [18] is valid only for so-called strictly hyperbolic polynomials, namely those where the roots $\lambda_{j}(u)$ are pairwise distinct when $u \neq 0$.

What about the other inequalities listed by A. Horn for $\operatorname{Det}_{\mathbf{H}_{n}}$ ? The high complexity of their proof in the Hermitian context, together with the lack of structure for general hyperbolic polynomials, discourage anyone to find a direct proof in the latter framework. Even their validity is far from obvious. We shall explain below that all of them actually do occur. To begin with, we recall P. Lax's conjecture that every homogeneous hyperbolic polynomial in three variables $P\left(\xi_{0}, \xi_{1}, \xi_{2}\right)$ (say that $P$ is hyperbolic in the direction $(1,0,0)$ ) can be written in the form

$$
\operatorname{det}\left(\xi_{0} A_{0}+\xi_{1} A_{1}+\xi_{2} A_{2}\right)
$$

for some Hermitian matrices $A_{0}, A_{1}, A_{2}$, with $A_{0}$ being positive definite. As remarked by $\mathrm{A}$. Lewis \& coll. [16], Lax's conjecture follows from a deep result about Riemann surfaces by J. W. Helton and V. Vinnikov [8]. Thus it has become a theorem, which can be applied for instance to the restriction of a given hyperbolic polynomial over $V$ to the subspace spanned by the elements $u, v$ and $e$. This restriction is still a hyperbolic polynomial, though in three variables only, and its roots are the restriction of the functions $\lambda_{i}$ 's. From the Hermitian case, we know that all of the Horn inequalities apply to these restrictions. Therefore the vectors $\lambda(u), \lambda(v)$ and $\lambda(u+v)$ satisfy the same inequalities. Whence:

Theorem 1.1 The inequalities listed by A. Horn are valid for the roots of every homogeneous hyperbolic polynomial.

We did not find a reference for this theorem, which is hardly a new result. According to Lewis, the validity of Lidskiı̌'s Theorem for every hyperbolic polynomial was remarked by L. Gurvits in 2004, who used the same argument as above. Although he did not mention such consequences in his paper, P. Lax could have had something like that in mind when he made his conjecture, even though only Weyl and Lidskiı-Wielandt inequalities were known at that time.

For readers interested in the conjecture of Lax, we recall that its $n$-dimensional counterpart becomes false for $n \geq 4$; for instance, it is impossible to find Hermitian matrices $A, \ldots, D$ with $A$ positive definite, such that

$$
X_{1}^{2}-X_{2}^{2}-X_{3}^{2}-X_{4}^{2}=\operatorname{det}\left(X_{1} A+X_{2} B+X_{3} C+X_{4} D\right) .
$$

Other inequalities. We point out that interesting nonlinear inequalities do occur in the theory of hyperbolic polynomials. For instance, Gårding [6] found the important fact that the restriction of $P^{1 / n}$ to its forward cone $\Gamma_{P}$ is concave. In terms of the roots, this means

$$
\left(\prod_{j=1}^{n} \lambda_{j}(u+v)\right)^{1 / n} \geq\left(\prod_{j=1}^{n} \lambda_{j}(u)\right)^{1 / n}+\left(\prod_{j=1}^{n} \lambda_{j}(v)\right)^{1 / n},
$$

whenever $\lambda_{1}(u)$ and $\lambda_{1}(v)$ are non-negative. This inequality has sound implications in geometry (Brunn-Minkowski inequality), combinatorics (Alexandrov-Fenchel and van der Waerden 
inequalities) or elliptic (!!) PDEs (boundary value problems for generalized Monge-Ampère equations).

A special case of (9) concerns the determinant of positive definite Hermitian matrices. The corresponding inequality

$$
(\operatorname{det}(A+B))^{1 / n} \geq(\operatorname{det} A)^{1 / n}+(\operatorname{det} B)^{1 / n}
$$

is strictly better than the well-known concavity of $A \mapsto \log \operatorname{det} A$. However, this latter property is the best one to be independent of the size $n$. As such, it has an extension to suitable Hermitian operators over Hilbert spaces.

\subsection{Four series of admissible triples $(I, J, K)$}

The theorems listed below follow directly from Theorem 1.1. We emphasize on their contents only because we shall give an elementary proof of them, which follows Weinberger's idea. Doing this, we avoid more involved tools, like cohomology of Riemann surfaces (Helton-Vinnikov's paper) and Schubert calculus. Having a direct and elementary proof has also the advantage that one can show that some triplets $(I, J, K)$ satisfy an inequality

$$
\Lambda_{K}(u+v) \geq \Lambda_{I}(u)+\Lambda_{J}(v), \quad \forall u, v \in V
$$

for every hyperbolic polynomial, without checking whether $(I, J, K) \in T_{r}^{n}$. On the one hand, the latter problem is NP-hard in terms of the degree $n$, and on the other hand, it might happen that $(I, J, K)$ belongs to $H_{r}^{n}$, though not to $T_{r}^{n}$.

We shall develop in the sequel a technique that yields a sufficient condition for an inequality of the form (5) to be valid for every hyperbolic polynomial. This condition has a combinatorial nature. It is not versatile enough to give back the whole Horn list. However, our technique is efficient enough to give the inequalities of Weyl and of Lidskiu, together with some others. One weakness of our method is that our condition depends on $n$, despite the fact that an inequality valid for some degree $n$ remains valid for a higher degree $N$. Here is Weyl's type inequality:

Theorem 1.2 Let $P$ be a hyperbolic polynomial of degree $n$ over $V$, with roots $\lambda_{1} \leq \cdots \leq \lambda_{n}$. If $1 \leq i, j, k \leq n$ satisfy $k+1=i+j$, then we have

$$
\lambda_{k}(u+v) \geq \lambda_{i}(u)+\lambda_{j}(v), \quad \forall u, v \in V
$$

Recall that the case $j=1, k=i$ was proved by Lax and Weinberger. Then we have the analogue of Lidskiı̌-Wielandt:

Theorem 1.3 Let $I \subset\{1, \ldots, n\}$ have cardinality $r, r<n$. Then we have

$$
\Lambda_{I}(u+v) \geq \Lambda_{I}(u)+\Lambda_{\min _{r}}(v), \quad \forall u, v \in V .
$$

Again, Lax and Weinberger's result is the case $r=1$. Now a sub-Lidskiu case, meaning that we replace the minimal sequence $(1, \ldots, r)$ by the sub-minimal one $(1, \ldots, r-1, r+1)$ : 
Theorem 1.4 Let $i_{1}<\cdots<i_{r}$ and $k_{1}<\cdots<k_{r}$ be such that $k_{p}=i_{p}$, except for one index $q$, for which $k_{q}=i_{q}+1$. Then we have

$$
\Lambda_{K}(u+v) \geq \Lambda_{I}(u)+\Lambda_{\operatorname{submin}_{r}}(v), \quad \forall u, v \in V .
$$

The last inequality of general breadth that we carry out is when $J=\pi I$. Then the optimality condition (7) implies that $K=\max$.

Theorem 1.5 Given $I \in \mathcal{I}_{r}^{n}$, the roots of an arbitrary hyperbolic polynomial of degree $n$ satisfy

$$
\Lambda_{\max _{r}}(u+v) \geq \Lambda_{I}(u)+\Lambda_{\pi I}(v), \quad \forall u, v \in V .
$$

Theorem 1.5 must have been known for a long time for Hermitian matrices. On the one hand, Inequality (14) is a rather natural generalization of Weyl's

$$
\lambda_{n}(A+B) \geq \lambda_{i}(A)+\lambda_{n+1-r}(B) .
$$

On the other hand, it is equivalent to (12), taking in account the relation $\Lambda_{I}(-u)=-\Lambda_{\pi I}(u)$; just apply $(12)$ to $(u,-u-v)$ instead of $(u, v)$. However, we shall give a direct proof of the Theorem 1.5. The same argument, applied to Theorem 1.4, gives the following:

Theorem 1.6 Let $i_{1}<\cdots<i_{r}$ and $j_{1}<\cdots<j_{r}$ be such that $j_{p}+i_{p}=n+1$ (that is $j_{p}=\pi i_{p}$ ), except for one index $q$, for which $j_{q}+i_{q}=n$. Then we have

$$
\Lambda_{\operatorname{submax}_{r}}(u+v) \geq \Lambda_{I}(u)+\Lambda_{J}(v), \quad \forall u, v \in V .
$$

By-products. Let $d$ be the g.c.d. of $n$ and $r$. Given $I$ in $\mathcal{I}_{r}^{n}$, it is possible to find $n / d$ sets $I_{\ell}$ in $\mathcal{I}_{r}^{n}$ such that the disjoint union of the $I_{\ell}$ 's be identical to the disjoint union of $r / d$ copies of $\{1, \ldots, n\}$, while $I_{1}=I$. Since each of the numbers

$$
\Lambda_{I_{\ell}}(u+v)-\Lambda_{I_{\ell}}(u)-\Lambda_{\min _{r}}(v)
$$

is non-negative, each one is less than the sum of all of them. This gives the majorization

$$
\Lambda_{I}(u+v)-\Lambda_{I}(u)-\Lambda_{\min _{r}}(v) \leq \frac{r}{d} \operatorname{Tr}(v)-\frac{n}{d} \Lambda_{\min _{r}}(v) .
$$

In other words, we have

$$
\Lambda_{I}(u+v) \leq \Lambda_{I}(u)+\frac{d+r-n}{d} \Lambda_{\min _{r}}(v)+\frac{r}{d} \Lambda_{\max _{n-r}}(v) .
$$

Depending on the pair $(n, r)$, (16) may or may not follow directly from the Lidskiu-Wielandt inequality

$$
\Lambda_{I}(u+v) \leq \Lambda_{I}(u)+\Lambda_{\max _{r}}(v) .
$$

Likewise, since each of the numbers

$$
\Lambda_{\max _{r}}(u+v)-\Lambda_{I_{\ell}}(u)-\Lambda_{\pi I_{\ell}}(v)
$$


is non-negative, each one is less than the sum of all of them. This gives the majorization

$$
\Lambda_{\max _{r}}(u+v)-\Lambda_{I}(u)-\Lambda_{\pi I}(v) \leq \frac{n}{d} \Lambda_{\max _{r}}(u+v)-\frac{r}{d} \operatorname{Tr}(u+v),
$$

whence the inequality

$$
(n-d) \Lambda_{\max _{r}}(u+v) \geq r\left(\Lambda_{I}(u)+\Lambda_{\pi I}(v)\right)+(r-d)\left(\Lambda_{I}(u)+\Lambda_{\pi I}(v)\right) .
$$

Once again, (17) follows directly or not from Theorem 1.3, depending on the pair $(n, r)$.

\section{An algebraic fact about hyperbolic polynomials}

The following auxiliary result is taken from our book with S. Benzoni-Gavage [1], Section 1.4.1.

Let $P$ be a hyperbolic polynomial of degree $n$ over $V$. Given two vectors $u, v \in V$, we define a polynomial

$$
R(X, Y):=P(X e-u-Y v),
$$

which has partial degrees $n$ with respect to $X$ and at most $n$ with respect to $Y$.

Proposition 2.1 Let $\left(\lambda^{*}, \mu^{*}\right)$ be a root of $R$. Then the multiplicity $N$ of $\lambda^{*}$ as a root of $R\left(\cdot, \mu^{*}\right)$, and the multiplicity $M$ of $\mu^{*}$ as a root of $R\left(\lambda^{*}, \cdot\right)$, satisfy

$$
N \leq M
$$

\section{Proof}

Up to a translation, we may assume that $\lambda^{*}=\mu^{*}=0$. We consider the Newton polygon of $R$, which is the lower convex hull of integral points $(l, m) \in \mathbb{N}^{2}$ associated to monomials $X^{l} Y^{m}$ present in $R$. By assumption, the Newton's polygon of $R$ admits the vertices $(N, 0)$ and $(0, M)$.

Let $\delta$ be the edge of the Newton's polygon with vertex $(N, 0)$. We denote its other vertex by $(j, k)$. The monomials of $P$ whose degrees $(a, b)$ belong to $\delta$ have the form $\operatorname{cst} \cdot X^{p} Y^{q}$ with $p=(1-\theta) N+\theta j$ and $q=\theta k$ for some $\theta \in[0,1]$. When we substitute $X \mapsto a^{k} X$ and $Y \mapsto a^{N-j} Y$, such a monomial is multiplied by $a^{k N}$. Therefore the sum of all such monomials of $P$ has the form $X^{j} Q$, where the polynomial $Q$ has the following homogeneity:

$$
Q\left(a^{k} X, a^{N-j} Y\right)=a^{k(N-j)} Q(X, Y) .
$$

It is a basic fact in algebraic geometry (see [3], Section 2.8) that, in the vicinity of the origin, the algebraic curve $R(x, y)=0$ is described by simpler curves corresponding to the edges of the Newton polygon, up to analytic diffeomorphisms. In the present case, these diffeomorphisms have real coefficients (i.e. they preserve real vectors) since $P$ has real coefficients. The "simple" curve $\gamma$ associated to $\delta$ is just that with equation $Q(x, y)=0$. Hence points $(x, y)$ in $\gamma$ with a real coordinate $y$ must be real (because this is so in the curve $P=0$.)

Let $\omega$ be a root of unity, of order $2(N-j)$, that is $\omega^{N-j}=-1$. Because of the homogeneity, the map $(x, y) \mapsto\left(\omega^{k} x,-y\right)$ preserves $\gamma$. If $y$ is real, the map thus moves a real point into 
an other one. Hence $\omega^{k}$ is real, thus $\omega^{2 k}=1$. This implies that $k$ is a multiple of $N-j$. In particular, $k \geq N-j$.

Since $(j, k)$ is a vertex of the Newton polygon, lying between the vertices $(N, 0)$ and $(0, M)$, we have

$$
\frac{j}{N}+\frac{k}{M} \leq 1
$$

Together with $k \geq N-j$, this implies $M \geq N$.

The equality case. Let $\rho$ denote $k /(N-j)$, which is a positive integer. Then we find $\rho N \leq M$. In the equality case $M=N$, we thus have $\rho=1$ and $j+k=N$. Therefore the Newton's polygon has only two vertices $(N, 0)$ and $(0, N)$.

\section{A combinatorial criterion}

This section is the core of the article. We prove Theorem 3.1 below, from which Theorems 1.2 through 1.6 follow. Because the roots $\lambda_{i}$ depend continously on the polynomial itself, an inequality of the form (10) is valid for every hyperbolic polynomial of degree $n$ if, and only if, it is valid for a polynomial in some dense subset of the class of hyperbolic polynomials in the direction $e$.

Because of Propositions 4.2 and 4.3, we may thus restrict to polynomials $P$ with the property that, for each $1 \leq r \leq n$, there exists an algebraic subvariety $\mathcal{M}_{r}$ of $\mathbb{R}^{d}$ (a strict one: $\mathcal{M}_{r} \neq \mathbb{R}^{d}$ ), such that the functions $\Lambda_{I}$ are pairwise distinct everywhere in $\mathcal{O}_{r}$, the complement of $\mathcal{M}_{r}$, as $I$ runs over $\mathcal{I}_{r}^{n}$.

Thus let $P$ be such a hyperbolic polynomial. We denote by $\mathcal{O}$ the intersection of the $\mathcal{O}_{r}$ 's, a dense open subset of $\mathbb{R}^{d}$. To prove that the inequality

$$
\Lambda_{K}(u+v) \geq \Lambda_{I}(u)+\Lambda_{J}(v)
$$

holds for every $u, v \in \mathbb{R}^{d}$, it is therefore enough to consider the case when $u, v$ and $u+v$ belong to $\mathcal{O}$; this now because the roots $\lambda_{i}(v)$ depend continously on $v$. In the sequel, we shall need actually only $v \in \mathcal{O}$.

Following Weinberger [18], we proceed by contradiction. Given $I, J, K \in \mathcal{I}_{r}^{n}$, we thus assume that

$$
\Lambda_{K}(u+v)<\Lambda_{I}(u)+\Lambda_{J}(v)
$$

holds true for some $u, v$ in $V$, with $v \in \mathcal{O}$. Let $t \in \mathbb{R}$ be given, and $v^{\prime}$ defined by $v^{\prime}=v-t e$. Then

$$
\Lambda_{I}(u)-\Lambda_{K}\left(u+v^{\prime}\right)=\Lambda_{I}(u)-\Lambda_{K}(u+v)+r t=\eta-\Lambda_{J}\left(v^{\prime}\right),
$$

where

$$
\eta:=\Lambda_{J}(v)+\Lambda_{I}(u)-\Lambda_{K}(u+v)
$$


is positive by assumption. Let us choose $t$ such that $0<\Lambda_{J}(v-t e)<\eta$. Then, dropping the prime, we are lead to the situation where we have

$$
\Lambda_{K}(u+v)<\Lambda_{I}(u), \quad \Lambda_{J}(v)>0 .
$$

This argument allows us to make $\Lambda_{J}(v)$ as small as desired. Since it does not change the differences $\lambda_{j+1}-\lambda_{j}$, and since the $\Lambda_{R}(v)$ 's are pairwise distinct, we may assume in addition that

$$
\left(R, S \in \mathcal{I}_{r}^{n}, R \neq S\right) \Longrightarrow\left(\left|\Lambda_{R}(v)-\Lambda_{S}(v)\right|>\Lambda_{J}(v)\right)
$$

In particular, we see that $\Lambda_{R}(v) \neq 0$ for every $R \in \mathcal{I}_{r}^{n}$, and

$$
(R \prec J, R \neq J) \Longrightarrow\left(\Lambda_{R}(v)<0\right) .
$$

We now choose a number $\lambda^{*}$ in the interval $\left(\Lambda_{K}(u+v), \Lambda_{I}(u)\right)$. Given $R$ in $\mathcal{I}_{r}^{n}$, we define the continuous function

$$
\mu \mapsto \phi_{R}(\mu):=\Lambda_{R}(u+\mu v),
$$

and look for a lower bound $m_{R}$ of the number of roots $\mu$ of the equation

$$
\phi_{R}(\mu)=\lambda^{*}
$$

Whenever $(R, \mu)$ satisfies $(22)$, one has

$$
P^{(r)}\left(\frac{1}{r} \lambda^{*} e-u-\mu v\right)=0 .
$$

Let $m:=\sum\left\{m_{R} ; R \in \mathcal{I}_{r}^{n}\right\}$. If the $m$ pairs $(R, \mu)$ have pairwise distinct $\mu$ 's, then the polynomial

$$
X \mapsto P^{*}(X):=P^{(r)}\left(\frac{1}{r} \lambda^{*} e-u-X v\right)
$$

has $m$ roots (actually $m$ real roots) at least. However, it may happen that a $\mu$ occurs in several pairs $(R, \mu)$. Let $a_{\mu}$ be the number of such pairs, for each $\mu$. We have

$$
m=\sum\left\{a_{\mu} ; \mu \in \mathbb{R}\right\} .
$$

By definition, $\lambda^{*} / r$ is a root of multiplicity $a_{\mu}$ of the polynomial

$$
Y \mapsto P^{(r)}(Y e-u-\mu v)
$$

Proposition 2.1 tells us that $\mu$ is a root of multiplicity at least $a_{\mu}$ of the polynomial $P^{*}$. We thus deduce that $P^{*}$ admits at least $m$ real roots, counting multiplicities.

To end with a contradiction, it will thus be enough to prove that

$$
m>\left(\begin{array}{l}
n \\
r
\end{array}\right)
$$


the right-hand side being the degree of $P^{*}$. With such a contradiction, we shall have proven the validity of

$$
\Lambda_{K}(u+v) \geq \Lambda_{I}(u)+\Lambda_{J}(v), \quad \forall u, v \in V .
$$

It thus remains to find the lower bounds $m_{R}$, and to sum them. Each $m_{R}$ will be obtained from the variations of $\phi_{R}$, with the help of the Intermediate Value Theorem. We shall use the values of $\phi_{R}$ at $\mu=0$ (where it equals $\Lambda_{R}(u)$ ) and at $\mu=1$ (where it equals $\Lambda_{R}(u+v)$ ), as well as its behaviour at infinity, which is governed by the sign of $\mu$ and that of either $\Lambda_{R}(v)$ or $\Lambda_{\pi R}(v)$.

At $\mu=0$, we can only say that

$$
(R \succ I) \Longrightarrow\left(\Lambda_{R}(u)>\lambda^{*}\right)
$$

Likewise, at $\mu=1$, we have

$$
(R \prec K) \Longrightarrow\left(\Lambda_{R}(u)<\lambda^{*}\right) .
$$

To investigate the behaviour of the $\phi_{R}$ 's at infinity, we need to split $\mathcal{I}_{r}^{n}$ into $\mathcal{P}$, the set of parts such that $\Lambda_{R}(v)<0$, and its complement $\mathcal{P}^{c}$, for which $\Lambda_{R}(v)<0$. We recall that none of the numbers $\Lambda_{R}(v)$ vanish. Although we do not know explicitly $\mathcal{P}$, we can state a few properties that it must satisfy. To begin with, we have $[J, \max ] \subset \mathcal{P}$ and $[\min , J) \subset \mathcal{P}^{c}$. If $R \prec S$ and $R \in \mathcal{P}$ (resp. $S \in \mathcal{P}^{c}$ ), then $S \in \mathcal{P}$ (resp. $R \in \mathcal{P}^{c}$ ). Mind however that these observations do not give a complete description of $\mathcal{P}$. There remains much freedom, and we shall have to deal with the worst possible case along our analysis.

Given $R$ in $\mathcal{I}_{r}^{n}$, the behaviour at $\pm \infty$ of $\phi_{R}$ is given by

$$
\phi_{R}(\mu) \sim \mu \times \begin{cases}\Lambda_{R}(v), & \mu \rightarrow+\infty \\ \Lambda_{\pi R}(v), & \mu \rightarrow-\infty\end{cases}
$$

Therefore $\phi_{R}$ is positive or negative at $+\infty$ (resp. $-\infty$ ) depending on whether $R$ belongs to $\mathcal{P}$ or $\mathcal{P}^{c}$ (resp. to $\pi \mathcal{P}$ or to $\pi \mathcal{P}^{c}$ ).

The simplest situation is when $R \in \mathcal{P}^{c} \cap \pi \mathcal{P}^{c}$, for then $\phi_{R}( \pm \infty)=\mp \infty$. Then there must exist one root : $m_{R}=1$.

If $R \in \mathcal{P} \cap \pi \mathcal{P}^{c}$, then $\phi_{R}( \pm \infty)=+\infty$. We can get a conclusion if moreover $R \in\left[\min _{r}, K\right]$, because then $\phi_{R}(1)<\lambda^{*}$. There must exist at least two roots : $m_{R}=2$.

Likewise, if $R \in \mathcal{P}^{c} \cap \pi \mathcal{P} \cap\left[I, \max _{r}\right]$, then $\phi_{R}( \pm \infty)=-\infty$ and $\phi_{R}(0)>\lambda^{*}$. Again, $m_{R}=2$.

If $R \in \mathcal{P} \cap \pi \mathcal{P}$, the situation is slightly more elaborate. Since there holds $\phi_{R}( \pm \infty)= \pm \infty$, we have $m_{R}=1$ at least. However, if moreover $R \in[I, K]$, then we have $\phi_{R}(1)<\lambda^{*}<\phi_{R}(0)$, and there are at least three roots : $m_{R}=3$.

The above analysis yields the following lower bound of the number of roots of $P^{*}$, where the bars stand for the cardinal of finite sets :

$$
\begin{aligned}
m=m(\mathcal{P})= & \left|\mathcal{P}^{c} \cap \pi \mathcal{P}^{c}\right|+|\mathcal{P} \cap \pi \mathcal{P}| \\
& +2\left(\left|\mathcal{P} \cap \pi \mathcal{P}^{c} \cap\left[\min _{r}, K\right]\right|+\left|\mathcal{P}^{c} \cap \pi \mathcal{P} \cap\left[I, \max _{r}\right]\right|+|\mathcal{P} \cap \pi \mathcal{P} \cap[I, K]|\right)
\end{aligned}
$$


The notation emphasizes that the bound $m(\mathcal{P})$ depends on $\mathcal{P}$, a set which, as said above, is not exactly known. Thus an explicit lower bound is given by the infimum $e(I, J, K)$ of $m(\mathcal{P})$ as $\mathcal{P}$ runs over all the subsets of $\mathcal{I}_{r}^{n}$ which satisfy the requirements already mentionned. The determination of $e(I, J, K)$ is a combinatorial problem that can be solved in finite time. When $e(I, J, K)$ is strictly larger than the degree of $P^{(r)}$, we have our contradiction, since the number of the roots of the univariate polynomial $P^{*}$, counting with multiplicities, would be larger than the degree. Therefore we can state our sufficient condition:

Theorem 3.1 Let $1 \leq r \leq n$ be two integers and $I, J, K$ be given in $\mathcal{I}_{r}^{n}$. If

$$
e(I, J, K)>\left(\begin{array}{c}
n \\
r
\end{array}\right)
$$

then the inequality (24) is valid for every hyperbolic polynomial of degree $n$.

\section{Density results}

The following statement is taken from [1], Section 1.5.4.

Lemma 4.1 Let $P\left(X ; \theta_{1}, \cdots, \theta_{d}\right)$ be a homogeneous polynomial of degree $n$ in $1+d$ variables, with real coefficients. Assume that the coefficient of $X^{n}$ is non-zero. Assume also that for all $\theta$ in a non-void open subset $\mathcal{O}$ of $\mathbb{R}^{d}$, the polynomial $P_{\theta}:=P(\cdot, \theta)$ has a root with multiplicity $\geq 2$. Then $P$ is reducible in $\mathbb{R}[X, \theta]$.

\section{Proof.}

Let us denote by $R:=\mathbb{R}\left[\theta_{1}, \cdots, \theta_{d}\right]$ the factorial ring of polynomials in $d$ variables $\theta$ and by $k:=\mathbb{R}\left(\theta_{1}, \cdots, \theta_{d}\right)$ the field of rational fractions in $\theta$. We first consider $P$ as an element of $k[X]$. Let us recall that $k[X]$ is a Euclidean ring, which has therefore a g.c.d.

Let $Q$ be the g.c.d of $P$ and $P^{\prime}$ in $k[X]$, a monic polynomial of $X$. Its coefficients, belonging to $k$, are rational fractions of $\theta$. We denote by $Z$ the zero set of the product of denominators of these fractions ; $Z$ is a closed set with empty interior.

When $\theta \in \mathcal{O} \backslash Z$ (this is a non-void open set), $Q_{\theta}:=Q(\cdot, \theta)$ has a non-trivial root, which means that either $Q_{\theta} \equiv 0$ or $d^{\circ} Q_{\theta} \geq 1$. However, the condition $Q_{\theta} \equiv 0$ defines a non-trivial algebraic manifold $M$ (the intersection of the zero sets of the coefficients of $Q$ ), again a closed set with empty interior. Therefore, there exists a $\theta$ for which $d^{\circ} Q_{\theta} \geq 1$, and consequently $d_{X}^{\circ} Q \geq 1$.

Since $Q$ divides $P$ in $k[X]$, we write $P=Q T$, with $T \in k[X]$. Multiplying by the l.c.m. of the denominators of all coefficients of $Q$ and $T$ (a l.c.m. and a g.c.d. do exist in the factorial ring $R$ ), we have $g(\theta) P=Q_{1} T_{1}$, where $g \in A, Q_{1}, T_{1} \in R[X]$ and $0<d_{X}^{\circ} Q_{1}<n$. We recall that the contents of a polynomial $S \in R[X]$, denoted by $c(S)$, is the g.c.d. of all its coefficients. From Gauss' Lemma, $c\left(Q_{1} T_{1}\right)=c\left(Q_{1}\right) c\left(T_{1}\right)$ and therefore $g=c\left(Q_{1}\right) c\left(T_{1}\right)$, since $c(P)=1$ by assumption. We conclude that $P=Q_{2} T_{2}$, where $Q_{2}:=c\left(Q_{1}\right)^{-1} Q_{1} \in R[X]$ and similarly $R_{2} \in R[X]$. Moreover $0<d_{X}^{\circ} Q_{2}<n$, which shows that $P$ is reducible in $R[X]=\mathbb{R}[X, \theta]$. 
Let $P$ be a homogeneous polynomial, hyperbolic in a direction e. Let us write $P$ as a product of irreducible factors

$$
P=\prod_{k=1}^{r} P_{k}^{\alpha(k)},
$$

where the $P_{k}$ 's are pairwise distinct. Each $P_{k}$ is homogeneous and hyperbolic in the direction e. The polynomial $X \mapsto P_{k}(X \mathbf{e}-\xi)$ has distinct roots away from the zero set $\mathcal{V}_{k}$ of its discriminant $D_{k}(\xi)$. Since $D_{k}$ is a polynomial, $\mathcal{V}_{k}$ is either the whole $\mathbb{R}^{d}$ or a codimension-1 algebraic variety. From Lemma 4.1, the latter case holds true. Away from $\mathcal{V}:=\bigcup_{k} \mathcal{V}_{k}$, the roots of $X \mapsto P(X \mathbf{e}-\xi)$ are of constant multiplicities $\alpha(k)$.

In particular, we have the following property.

Proposition 4.1 Let $P$ be a homogeneous hyperbolic polynomial. Assume that $P$ is square-free (that is, $P$ does not have a factor $Q^{2}$ where $Q$ is a non-constant polynomial). Then the roots of $X \mapsto P(X \mathbf{e}-\xi)$ are simple for every $\xi \in \mathcal{O}$, an open dense set in $\mathbb{R}^{d}$.

We now show that square-free polynomials are dense within hyperbolic ones. We proceed by induction on $\alpha(P)$, the largest of the $\alpha(k)$ 's in the factorization above. Because each $P_{k}$ in the factorization above is hyperbolic in the same direction $\mathbf{e}$, it is enough to assume that $P=p^{\alpha}$, where $p$ is irreducible. It will be enough to consider the case of a square $p^{2}$, since then we may write

$$
P=p^{\alpha-2} p^{2}, \quad p^{2}=\lim p_{\ell},
$$

where $p_{\ell}$ is e-hyperbolic and square-free. The primitive factors $P_{j}^{\beta}$ of $p^{\alpha-2} p_{\ell}$ have degree less than that of $P$. The induction assumption ensures thus that $p^{\alpha-2} p_{\ell}$ is approximated by squarefree hyperbolic polynomials. By the diagonal procedure, it is also true for $P$.

There remains therefore the case $P=p^{2}$ with $p$ irreducible. We recall that if $R(t)$ is a univariate polynomial with real roots, then

$$
R_{\gamma}(t):=R(t)+\gamma R^{\prime}(t)=e^{-\gamma t}\left(e^{\gamma t} R\right)^{\prime} .
$$

has real roots too, for every $\gamma \in \mathbb{R}$. Let $f$ be a linear form, vanishing at $\mathbf{e}$; we may therefore approximate $P$ by the homogenous polynomials

$$
P_{\epsilon}(v):=P(v)+\epsilon f(v) D P(v) \cdot \mathbf{e},
$$

where we denote

$$
D P(v) \cdot e:=\left.\frac{d}{d t}\right|_{t=0} P(t \mathbf{e}+v) .
$$

Since

$$
P_{\epsilon}(t \mathbf{e}-v)=P(t \mathbf{e}-v)-\epsilon f(v) D P(t \mathbf{e}-v) \cdot \mathbf{e}
$$

is of the form $R_{\gamma}$, with $\gamma=\epsilon f(v)$, the equation $P_{\epsilon}(t \mathbf{e}-v)=0$ has only real roots: $P_{\epsilon}$ is hyperbolic. Of course, $P_{\epsilon}$ is divisible by $p$, but not by $p^{2}$. Then we conclude with the induction assumption.

We have thus proven: 
Proposition 4.2 The set of square-free homogeneous polynomials, hyperbolic in direction $\mathbf{e}$, is open and dense in the set of homogeneous polynomials, hyperbolic in direction $\mathbf{e}$. For such polynomials, the $\lambda_{j}$ 's are pairwise distinct over a dense open subset $\mathcal{O}_{1}$ of $\mathbb{R}^{d}$.

We now consider the sums $\Lambda_{I}$ when $I$ runs over $\mathcal{I}_{r}^{n}$, and ask ourselves whether they are distinct over a dense open subset of $\mathbb{R}^{d}$.

Let $P$ be a polynomial as in Proposition 4.2. Let us fix a vector $v \in \mathcal{O}_{1}$. Since e is an interior point of $\Gamma_{P}$, the polynomial remains hyperbolic in the direction of $e_{\rho}:=\mathbf{e}-\rho v$ for every small enough $\rho \in \mathbb{R}$. Let us denote $\theta_{j}(\rho ; z)$ the roots of $P$ with respect to $e_{\rho}$ :

$$
P\left(\mu e_{\rho}-z\right)=0 \text {. }
$$

For a small $z$, we have the implicit relations

$$
\theta_{j}(\rho ; z)=\lambda_{j}\left(\theta_{j}(\rho ; z) \rho v+z\right)
$$

For $z=v$, this gives

$$
\theta_{j}(\rho ; v)=\frac{\lambda_{j}(v)}{1-\rho \lambda_{j}(v)}
$$

Let $I$ and $J$ be given, with the same cardinal and $I \neq J$. As far as we consider the difference $\Lambda_{I}-\Lambda_{J}$, we may assume that $I \cap J=\emptyset$, for otherwise we can replace them by $I^{\prime}=I \backslash(I \cap J)\left(J^{\prime}\right.$ defined likewise), with a smaller cardinal. Since the $\lambda_{j}(v)$ 's are pairwise distinct, the rational function

$$
\rho \mapsto \sum_{i \in I} \frac{\lambda_{i}(v)}{1-\rho \lambda_{i}(v)}-\sum_{j \in J} \frac{\lambda_{j}(v)}{1-\rho \lambda_{j}(v)}
$$

does not vanish identically. Its zero $\rho=0$ is thus isolated, and we deduce that for arbitrary small non-zero $\rho$, this difference is non null. For such a $\rho$, the $\Theta_{I}$ 's are thus pairwise distinct at $v$, and therefore on a dense open subset of $\mathbb{R}^{d}$. Let us now choose a linear map $w \mapsto M_{\rho} w$, close to the identity, such that $M_{\rho} \mathbf{e}=e_{\rho}$. Let us form the homogeneous polynomial

$$
P_{\rho}(w):=P\left(M_{\rho} w\right)
$$

which tend to $P$ as $\rho \rightarrow 0$. The roots of $P_{\rho}(\cdot \mathbf{e}-w)$ are the numbers $\mu_{i}\left(\rho, M_{\rho} w\right)$. Therefore $P_{\rho}$ is hyperbolic in the direction $\mathbf{e}$, and its $\Lambda_{I}$ 's are pairwise distinct at $v$. Finally, we have proved:

Proposition 4.3 In the set of homogeneous polynomials, hyperbolic in direction $\mathbf{e}$, the subset of polynomials for which the $\Lambda_{I}$ 's, with $I \in \mathcal{I}_{r}^{n}$, are pairwise distinct functions over a dense open subset of $\mathbb{R}^{d}$, is a dense subset.

\section{Calculations in specific cases}

\subsection{Weyl-type inequalities}

Set $r=1$ and $k=i+j-1$. Because $\mathcal{I}_{1}^{n}$ is totally ordered, $\mathcal{P}=[j, n]$ is exactly known. We thus have $\pi \mathcal{P}=[1, n+1-j], \mathcal{P}^{c}=[1, j-1]$ and $\pi \mathcal{P}^{c}=[n+2-j, n]$. By symmetry, we may 
assume that $j \leq i$, so that $j=k-i+1 \leq n-j+1$. The first line in the definition of $m(\mathcal{P})$ sums up to $n+2-2 j$. In the second one, the first term is $(\min (k, n+1-j)-i+1)^{+}$. One finds $e(I, J, K)=m(\mathcal{P})=n+2>n$, which satisfies (25) when $r=1$. Whence the Weyl-type inequalities, our Theorem 1.2.

\subsection{Lidskiǔ-type inequalities}

Set $J=\min _{r}$ and $K=I$. Here, the only possible $\mathcal{P}$ is $\mathcal{I}_{r}^{n}$ itself. We have $\pi \mathcal{P}=\mathcal{I}_{r}^{n}$, while $\mathcal{P}^{c}$ is void. A straightforward computation gives

$$
e(I, J, K)=m\left(\mathcal{I}_{r}^{n}\right)=\left|\mathcal{I}_{r}^{n}\right|+2|[I, K]|=\left(\begin{array}{c}
n \\
r
\end{array}\right)+2 .
$$

Again, Theorem 3.1 tells that the corresponding inequality (24) is valid for every hyperbolic polynomial of degree $n$. This is our Theorem 1.3.

\subsection{Sub-Lidskiı̌ inequalities}

Set $J=\operatorname{supmin}_{r}$ and $I, K$ as mentionned in Theorem 1.4. The interval $[I, K]$ consists in exactly two elements $I$ and $K$. Then $\mathcal{P}$ can be either $\mathcal{I}_{r}^{n}$ or [supmin, max]. Correspondingly, we have either

$$
\pi \mathcal{P}=\mathcal{I}_{r}^{n}, \quad \mathcal{P}^{c}=\emptyset, \quad \pi \mathcal{P}^{c}=\emptyset
$$

or

$$
\pi \mathcal{P}=[\min , \text { submax }], \quad \mathcal{P}^{c}=\{\min \}, \quad \pi \mathcal{P}^{c}=\{\max \} .
$$

In the first case, we have easily

$$
m=2|[I, K]|+\left|\mathcal{I}_{r}^{n}\right|=4+\left(\begin{array}{c}
n \\
r
\end{array}\right) .
$$

In the second one, we have

$$
m=2|[I, K]|+|[\operatorname{supmin}, \operatorname{submax}]|=4+\left(\begin{array}{c}
n \\
r
\end{array}\right)-2 .
$$

In both cases, $m(\mathcal{P})$ is strictly larger than the degree $\left|\mathcal{I}_{r}^{n}\right|$ and we have the desired contradiction.

\subsection{When $J=\pi I$}

When $J=\pi I$, we have $K=\max _{r}$ by the optimality condition (7). For every admissible $\mathcal{P}$, we have the following calculations

$$
\begin{aligned}
m(\mathcal{P}) & =|\mathcal{P} \cap \pi \mathcal{P}|+\left|\mathcal{P}^{c} \cap \pi \mathcal{P}^{c}\right|+2\left(\left|\mathcal{P} \cap \pi \mathcal{P}^{c}\right|+\mid \pi \mathcal{P} \cap\left[I, \max _{r}\right]\right) \\
& =|\mathcal{P}|+\left|\pi \mathcal{P}^{c}\right|+2\left|\pi \mathcal{P} \cap\left[I, \max _{r}\right]\right| \\
& =\left|\mathcal{I}_{r}^{n}\right|+2\left|\pi \mathcal{P} \cap\left[I, \max _{r}\right]\right| .
\end{aligned}
$$


Since $\mathcal{P}$ contains $\left[J, \max _{r}\right], \pi \mathcal{P}$ contains $\left[\min _{r}, \pi J\right]$. Using $\pi J=I$, we thus have

$$
m(\mathcal{P}) \geq\left|\mathcal{I}_{r}^{n}\right|+2\left|\left[\min _{r}, \pi J\right] \cap\left[I, \max _{r}\right]\right|=\left|\mathcal{I}_{r}^{n}\right|+2|[I, \pi J]=| \mathcal{I}_{r}^{n} \mid+2 .
$$

In conclusion, we have $e\left(I, \pi I, \max _{r}\right)=\left|\mathcal{I}_{r}^{n}\right|+2$, which is the contradiction we were looking for.

\subsection{Gap with Horn's inequalities}

Here we show that $e(I, J, K)$ does not exceed the degree $\left|\mathcal{I}_{r}^{n}\right|$ for some explicit Horn's triplet. For such triplets, our combinatorial approach is thus inefficient.

A Freede-Thompson case. When $i_{s}+j_{s}=k_{s}+s$ for every $s=1, \ldots, r$, the triplet $(I, J, K)$ is admissible, according to Freede \& Thompson. One of the simplest such triplet which does not belong to a Lidskiı̆ or to a super-Lidskiu case is $I=J=\{2,3\}$ with $K=\{3,4\}$ where we take $n=4$. This example actually can be treated by applying Theorem 3.1. However, if we set $n=5$ instead, then the strategy fails, because of the choice

$$
\mathcal{P}=[\{1,2\},\{1,5\}],
$$

for which we have

$$
m(\mathcal{P})=10=\left|\mathcal{I}_{2}^{5}\right|
$$

Another Freede-Thompson case. An other minimal example is $I=J=\{1,4\}, K=$ $\{1,6\}$, with $n=6$. In the combinatorial criterion, we must consider the choice

$$
\mathcal{P}=[\{1,2\},\{2,3\}]
$$

with three elements. We leave the reader verifying that this choice yields

$$
m(\mathcal{P})=15=\left|\mathcal{I}_{2}^{6}\right|
$$

and therefore we cannot apply Theorem 3.1.

This example is the first one in an infinite series, given by the following proposition, whose proof is left to the reader.

Proposition 5.1 Assume that $2 \leq b \leq c, a<c, b \leq \pi b$ and that $a \leq \pi b$ (the latter is a Horn constraint when $r=2$ ). Set $I=(a, c), J=(1, b)$ and $K=(a, b+c-2)$ (a Freed $\&$ Thompson triple, admissible according to Horn). Take $\mathcal{P}=\left[J, \max _{2}\right]$. Then

$$
m(\mathcal{P})=\left(\begin{array}{l}
n \\
2
\end{array}\right)+(b-1)(4-b) .
$$

In particular, if $b \geq 4$, then $e(I, J, K)$ is less than or equal to $\left|\mathcal{I}_{2}^{n}\right|$ and Theorem 3.1 does not apply. 
Acknowledgement: Although I began to think about this problem when I prepared my book on matrices (Springer-Verlag, GTM \# 216 (2002)) and the book with S. Benzoni-Gavage on multi-dimensional hyperbolic IBVP (Oxford Univ. Press (2006)), it is fair to say that I learnt a lot about hyperbolic polynomials during my stay in Mittag-Leffler Institute in autumn 2005. I keep a deep impression of the marvelous library there, and the warm working atmosphere. It is thus a great pleasure to thank S. Benzoni-Gavage for her fruitful collaboration and the Mittag-Leffler Institute for its hospitality.

I have benefited also from the sustained interest of Peter Lax on that work.

\section{References}

[1] S. Benzoni-Gavage, D. Serre. Multi-dimensional hyperbolic partial differential equations: First-order systems and applications. Oxford Mathematical Monographs, Oxford University Press (2006).

[2] R. Bhatia. Linear algebra to quantum cohomology: the story of A. Horn's inequalities. Amer. Math. Monthly, 108 (2001), pp 289-318.

[3] Shui-Nee Chow, J. K. Hale. Methods of bifurcation theory. Grundlehren der mathematischen Wissenschaften, Vol. 251. Springer-Verlag, Heidelberg (1982).

[4] Ky Fan. On a theorem of Weyl concerning eigenvalues of linear transformations. Proc. Nat. Acad. Sci., 35 (1949), pp 652-655.

[5] W. Fulton. Eigenvalues, invariant factors, highest weights, and Schubert calculus. Bull. of the A. M. S., 37 (2000), pp 209-250.

[6] L. Gårding. Linear hyperbolic partial differential equations with constant coefficients. Acta Math., 85 (1951), pp 1-62. An inequality for hyperbolic polynomials. J. Math. Mech., 8 (1959), pp 957-965.

[7] J. Harris. Algebraic geometry. A first course. Springer-Verlag (1999), New York.

[8] J. W. Helton, V. Vinnikov. Linear matrix inequality representation of sets. Unpublished, (2002).

[9] A. Horn. Eigenvalues of sums of Hermitian matrices. Pacific J. Math., 12 (1962), pp 225241.

[10] T. Kato. Perturbation theory for linear operators. Springer-Verlag (1980), New York.

[11] A. Klyachko. Stable bundles, representation theory and Hermitian operators. Selecta Math. (N. S.), 4 (1998), pp 419-445.

[12] A. Knutson, T. Tao. The honeycomb model of $G L_{n}(\mathbb{C})$ tensor products. I. Proof of the saturation conjecture. J. Amer. Math. Soc., 12 (1999), pp 1055-1090. 
[13] B. Kostant. Lie algebra cohomology and the generalized Borel-Weil theorem. Annals of Math., 74 (1961), pp 329-387.

[14] P. D. Lax. The multiplicity of eigenvalues. Bull. Amer. Math. Soc., 6 (1982), pp 213-214.

[15] P. D. Lax. Differential equations, difference equations and matrix theory. Comm. Pure Appl. Math., 11 (1958), pp 175-194.

[16] A. S. Lewis, P. A. Parrilo, M.V. Ramana. The Lax conjecture is true. Proceedings of the Amer. Math. Soc., 133 (2005), pp 2495-2499.

[17] B. V. Lidskil. The proper values of the sum and the product of symmetric matrices. Dokl. Akad. Nauk SSSR, 74 (1950), pp 769-772.

[18] H. F. Weinberger. Remark on the preceeding paper of Lax. Comm. Pure Appl. Math., 11 (1958), pp 195-196.

[19] H. Weyl. Das asymptotische Verteilungsgesetz der Eigenwerte lineare partieller Differentialgleichungen. Math. Ann., 71 (1912), pp 441-479.

[20] H. Wielandt. An extremum property of sums of eigenvalues. Proc. Amer. Math. Soc., 6 (1955), pp 106-110. 